\title{
ACUPUNTURA NA ESTÉTICA: UMA REVISÃO DE LITERATURA SOBRE OS BENEFÍCIOS E APLICAÇÕES
}

\author{
Bianca Clara Vieira ${ }^{1}$ \\ Emanuely dos Santos Silva² \\ Caroline Valente ${ }^{3}$
}

\begin{abstract}
Resumo: Muito utilizadas para o tratamento de patologias, as práticas da Medicina Tradicional Chinesa (MTC) também possuem resultados eficazes na área da estética. Baseada em suas teorias básicas: a teoria do Yin e Yang, dos Cinco Movimentos e dos Zang Fu (órgãos e vísceras), as práticas da MTC visam tratar as patologias e as queixas estéticas do paciente através da busca pelo equilíbrio energético do organismo. O presente trabalho possui como objetivo buscar na literatura informações pertinentes ao conhecimento das práticas da MTC na estética, descrever sobre as práticas, seus benefícios e identificar quais práticas são mais eficazes para cada queixa estética. Trata-se de uma revisão bibliográfica elaborada através de dados disponíveis na literatura entre os anos de 1985 a 2017 , foram abordadas no trabalho dez práticas da MTC. Conclui-se que o tratamento estético através das práticas da MTC possui bons resultados na estética e diversas vantagens por ser prático, pouco invasivo, baixo risco de alergia, raras contraindicações, além de serem práticas que visam equilibrar o organismo do paciente.
\end{abstract}

Palavras-chave: acupuntura; estética; acupuntura estética; medicina tradicional chinesa.

\section{ACUPUNCTURE IN AESTHETICS: A LITERATURE REVIEW ON THE BENEFITS AND APPLICATIONS}

\begin{abstract}
Much used for the treatment of pathologies, Tradicional Chinese Medicine (TCM) practices also have effective results in the area of aesthetics. Based on their basic theories: the theory of Yin and Yang, the five movements and the Zang Fu (organs and viscera), the practices of TMC aims to treat the patient's pathologies and aesthetic complaints through the search for the energy balance of the organism. The present work aims to search in the literature for information pertinent to the knowledge of TCM practices in aesthetics, to describe the practices and their benefits. It is a bibliographical review elaborated through data available in the literature between the years 1985 to 2017, was addressed in the work ten practices of the TCM. It is concluded that the aesthetic treatment through the practices of the TCM, has good aesthetic results and numerous advantages because they are little invasive practices, low risk of allergy, rare against indications, besides being practices that aim at balancing the patient's body.
\end{abstract}

Keywords: acupuncture; aesthetics; aesthetic acupuncture; traditional chinese medicine.

\section{Introdução}

A Medicina Tradicional Chinesa (MTC) nasceu na China a milhares de anos atrás, e além de ser muito utilizada pelos Chineses ela também conquistou outros lugares do mundo, sendo utilizada em diversos países e reconhecida pela Organização Mundial de Saúde (OMS).

\footnotetext{
${ }^{1}$ Biomédica. Departamento de Farmácia. Centro de Ciências da Saúde. Universidade Regional de Blumenau - FURB. Blumenau - SC. Email: bclaravieira@ hotmail.com

${ }^{2}$ Acadêmica do curso de graduação em Medicina. Universidade Estadual do Oeste do Paraná - UNIOESTE, Campus de Francisco Beltrão - PR. Email: emanuelysantos@ hotmail.com

${ }^{3}$ Farmacêutica. Acupunturista. Professora. Departamento de Ciências Naturais. Centro de Ciências Exatas e Naturais. Universidade Regional de Blumenau - FURB. Blumenau - SC. Email: carolvalente11@ gmail.com
} 
Com sua filosofia própria a MTC possui três teorias básicas: a teoria do Yin e Yang, dos Cinco Movimentos e dos Zang Fu (Órgãos e Vísceras), as quais devem sempre ser levadas em consideração para o diagnóstico e tratamento do paciente, visando o equilíbrio energético do organismo.

Para a MTC, o aparecimento de uma patologia ou até mesmo queixas estéticas, surgem no indivíduo devido à falta de equilíbrio energético no organismo, por esse motivo, a MTC trabalha para restabelecer esse equilíbrio.

Além de muito eficazes para o tratamento de patologias, as práticas da MTC vêm ganhando espaço na área da estética e conseguem obter bons resultados, por exemplo, para redução de gordura localizada, rugas e celulite.

A MTC é composta por diversos tipos de práticas diferentes, que aliás, em alguns casos podem ser utilizadas juntas nos tratamentos para conquistar resultados mais satisfatórios.

Nosso corpo possui diversos pontos de acupuntura, e é nesses pontos que as diferentes práticas da MTC vão atuar para obter os resultados esperados tanto no tratamento de patologias como no tratamento estético.

\section{Práticas da medicina tradicional chinesa na estética:}

\section{Acupuntura}

A acupuntura estética tem como objetivo promover equilíbrio energético no organismo, permitindo a renovação e a volta da elasticidade da pele (RINALDI, 2014).

O tratamento de acupuntura estética pode ser realizado para reduzir a ansiedade e o estresse, que em muitos casos são os fatores que provocam insatisfação com a estética (RINALDI, 2014).

O tratamento também pode ser executado com a aplicação de agulhas na área a ser tratada, em alguns casos o tratamento é acompanhado por outras práticas da MTC, como eletroacupuntura, moxaterapia, entre outras práticas, buscando um resultado mais satisfatório (RINALDI, 2014).

Benefícios do tratamento com acupuntura na estética:

- $\quad$ Redução de rugas da face;

- $\quad$ Redução de gordura localizada;

- $\quad$ Redução de celulite;

- $\quad$ Redução de flacidez;

- $\quad$ Emagrecimento. 


\section{Auriculoterapia}

A auriculoterapia visa estimular os pontos localizados no pavilhão auricular, que representam os órgãos e vísceras do nosso organismo (DOMINGO, 2011).

$\mathrm{Na}$ estética, a auriculoterapia é muito utilizada para auxiliar no emagrecimento. O tratamento é baseado em promover equilíbrio físico, energético e emocional. Através da utilização de sementes em pontos estratégicos localizados na orelha, a auriculoterapia diminui a ansiedade, desta forma, controlando a compulsão por comer (DOMINGO, 2011).

O fato isolado de estimular os pontos na orelha não irá fazer o paciente eliminar peso, o tratamento de auriculoterapia auxilia na ansiedade do paciente para que consiga se manter na dieta controlando a compulsão por comida, então o paciente irá conquistar bons resultados de emagrecimento se combinar a dieta com o tratamento de auriculoterapia (DOMINGO, 2011).

Benefícios do tratamento com acupuntura na estética:

- $\quad$ Emagrecimento.

Os pontos que serão utilizados no tratamento de auriculoterapia são escolhidos na primeira sessão, em seguida será feita uma higienização com álcool $75 \%$, após isso serão posicionadas as sementes em seus respectivos pontos e presas por fita microporosa. As sementes permanecem na orelha de forma indolor e permanecerão por 05 dias, após 05 dias serão retiradas (DOMINGO, 2011).

\section{Ventosaterapia}

Na estética, o tratamento com ventosa geralmente está aliado ao uso de óleos essenciais aromaterápicos utilizados para massagear o paciente. $\mathrm{O}$ procedimento com as ventosas utilizados na estética é diferente da técnica de aplicação direta das ventosas, pois a aplicação direta pode gerar, por exemplo, hematomas, e não seria bem visto após um procedimento estético. No caso da ventosa associada a óleos essenciais aromaterápicos, o tratamento estético conquista ótimos resultados após a ventosaterapia, a massagem de drenagem linfática na área onde foi aplicada as ventosas conquista resultados ainda mais satisfatórios (FORNAZIERI, 2007).

É indicado que as ventosas utilizadas na estética sejam de vidro chinês ou coreano com formato esférico, os copos tradicionais não são os mais apropriados para esse procedimento, pois pode ocorrer de o copo trincar e desta forma machucar o indivíduo que está fazendo o uso da ventosa (FORNAZIERI, 2007). 
Benefícios do tratamento com ventosas na estética:

- $\quad$ Tratamento de gordura localizada;

- $\quad$ Tratamento de celulite.

A ventosa é aplicada nas regiões do corpo que possui acúmulo de gordura localiza, ou em regiões de celulite, o óleo essencial aromático é sobreposto na área em que será aplicada a ventosa (FORNAZIERI, 2007).

Para o tratamento de celulite é realizada a técnica de deslizamento com ventosa e óleos essenciais, que tem como objetivo liberar energias estagnadas no local onde existe celulite, e assim, facilitando a circulação de Qi e Xue.

\section{Moxaterapia}

A moxaterapia tem como objetivo produzir calor, no procedimento é realizada a queima de ervas medicinais (sendo a mais utilizada a Artemísia), a qual é posicionada sobre os pontos desejados (FERNANDES, 2015).

Benefícios do tratamento com Moxa na estética:

- $\quad$ Tratamento de rugas;

- $\quad$ Produção de colágeno;

- $\quad$ Promove viçosidade a pele.

A moxa é conhecida em ser uma prática complementar às agulhas, porém é uma prática tão eficaz como a acupuntura feita com agulhas. Além da eficácia para terapêutica de dores, a moxaterapia conquista excelentes resultados para o tratamento de rugas da face (FERNANDES, 2015)

A atividade da moxa no organismo produz colágeno e aumenta a circulação de Qi e Xue, tornando a pele mais viçosa (FERNANDES, 2015). Segundo o autor, a moxa é contraindicada em padrões de Calor, Calor-Vazio, quadros de calor, indivíduo com febre, estrias vermelhas e também em lesões de pele.

Para Fernandes (2015), a aplicação da moxa é realizada com o bastão sustentado a uma distância da pele que gere um calor tolerável ao paciente, até gerar hiperemia. Deve-se ter cautela com indivíduos que possuem redução ou a total falta de sensibilidade periférica, como por exemplo, indivíduos com distúrbios neurológicos. 


\section{FAZ CIÊNCIA, VOL. 23, N. 37, JAN/JUN DE 2021 - P. 211 - 224}

\section{Gua-sha}

$\mathrm{Na}$ estética, o Gua-sha é aplicado para propiciar estímulo sem ser uma prática invasiva. O Gua-sha mais utilizado é feito de jade, e é usado na face auxiliando a drenagem de líquidos (JING YE) e promovendo a circulação de sangue (Xue) (FERNANDES, 2015).

Benefícios do tratamento com Gua-sha na estética:

- Produção de colágeno;

- Promove viscosidade a pele.

A aplicação do Gua-sha na face é realizada abaixo dos olhos, sentido asa do nariz à orelha e em cima das sobrancelhas, sentido sobrancelhas ao cabelo, fazendo o movimento por toda a testa (FERNANDES, 2015).

\section{Algas Marinhas}

Na estética podem ser utilizadas algumas espécies de algas verdes, também são utilizadas algas vermelhas (FERNANDES, 2015).

Segundo Fernandes (2015), para fazer uso das algas na estética as algas verdes devem ser hidratadas em água limpa e filtrada por 4 a 12 horas, e usadas trituradas em pouca quantidade de água, já as algas vermelhas trituradas tem seu uso mais facilitado, pois podem ser reidratadas em água em um curto tempo anterior a sua aplicação.

As algas são aplicadas na pele com pincel ou espátula e devem ser aplicadas em toda a área desejada. O tempo necessário de manter as algas verdes sobre a pele é em torno de 15 a 30 minutos, já as algas vermelhas podem permanecer na pele até secar totalmente. As algas verdes e vermelhas podem ser retiradas da pele com água morna (FERNANDES, 2015).

Benefícios da aplicação de algas marinhas na estética:

- $\quad$ Produção de colágeno;

- $\quad$ Hidratação da pele;

- $\quad$ Ameniza estrias.

\section{Pó de Perola}

O pó de pérola tem em sua composição cálcio, que possui atividade reestruturadora e revigorante, aminoácidos, que tem como benefício potencializar as atividades metabólicas e enzimáticas, e zinco, que tem como finalidade proteger a pele (FERNANDES, 2015). 


\section{FAZ CIÊNCIA, VOL. 23, N. 37, JAN/JUN DE 2021 - P. 211 - 224}

Os aminoácidos são fundamentais para produção de diversas proteínas com ação estrutural na pele, favorecendo nutrição, proteção, hidratação e efeito lifiting imediato. O pó de pérola possui absorção pela pele de 95 a 99\% (FERNANDES, 2015).

Segundo especialistas chineses, as pérolas podem reparar os estragos gerados a pele pelo sol. O pó de pérola possui proteínas que revitalizam as células da pele (FERNANDES, 2015). Benefícios do tratamento com pó de pérola na estética:

- $\quad$ Produção de colágeno;

- $\quad$ Hidratação da pele;

- $\quad$ Previne e ameniza manchas na pele.

\section{Laser}

Segundo Aiabe (2011), o objetivo do tratamento estético utilizando o laser é muito semelhante ao tratamento estético utilizando agulhas da acupuntura clássica, porém nesse caso os pontos de acupuntura serão estimulados pelo laser.

Benefícios do tratamento com acupuntura na estética:

- $\quad$ Redução de rugas da face;

- $\quad$ Redução de gordura localizada;

- $\quad$ Redução de celulite;

- $\quad$ Redução de flacidez;

- $\quad$ Emagrecimento.

O procedimento é realizado com um laser de baixa potência que estimula os pontos de acupuntura, as células do organismo do paciente absorvem a radiação luminosa conquistando resultados semelhantes ao da acupuntura com agulhas. O procedimento é indolor (AIABE, 2011).

\section{Eletroacupuntura}

$\mathrm{Na}$ eletroacupuntura são utilizadas agulhas da acupuntura clássica combinada com a eletroterapia, com a finalidade de estimular os pontos de acupuntura (FERNANDES, 2015; NAKANO e YAMAMURA, 2005).

Benefícios do tratamento com eletroacupuntura na estética:

- $\quad$ Tratamento de rugas da face;

- $\quad$ Tratamento de gordura localizada;

- $\quad$ Tratamento de celulite; 
- $\quad$ Tratamento de estrias da pele;

- $\quad$ Tratamento de flacidez.

Para o tratamento de eletroacupuntura nas rugas da face, é imprescindível que o profissional que está realizando o procedimento conheça a anatomia e as funções da musculatura da face, será necessário compreender as funções antagonista e agonista (NAKANO e YAMAMURA, 2005).

Para o procedimento de eletroacupuntura no tratamento de gordura localizada é necessário demarcar a região e existe duas formas de proceder o tratamento, a primeira forma é tratar a gordura localizada com pontos de dor fora dos meridianos, que nesse cenário seria a aplicação de agulhas de acupuntura em volta da região demarcada de gordura localizada e em seguida posicionar os eletrodos fazendo com que as correntes elétricas se cruzem entre a gordura localizada. A segunda forma é tratar a gordura localizada como se fosse um processo inflamatório, no qual o procedimento consiste em aplicação de agulhas ao redor da região demarcada de gordura localizada, e em seguida, é aplicada a mesma quantidade de agulhas na região central da gordura localizada e, posteriormente, é posicionado os eletrodos, este procedimento gera um estímulo que lisa o tecido adiposo, que será excretado pela urina. Para não gerar excesso a função renal, é necessário que o tratamento seja restrito durante a semana a apenas duas regiões do corpo (NAKANO e YAMAMURA, 2005).

Para o tratamento de celulite, o procedimento com eletroacupuntura, pode ocorrer em torno de 20 a 30 minutos, sendo estimuladas as áreas que estão presentes a celulite (NAKANO e YAMAMURA, 2005).

No tratamento de eletroacupuntura para estrias, as agulhas da acupuntura são aplicadas no percurso das estrias, e são estimuladas até gerar um pequeno edema e vermelhidão local. O procedimento de eletrocupuntura no tratamento de estrias pode gerar dor ao paciente (NAKANO e YAMAMURA, 2005).

Segundo Nakano e Yamamura (2005), o tratamento com esse método para flacidez deve buscar a harmonização dos Zang $\mathrm{Fu}$, fazendo uso dos pontos sistêmicos.

O tratamento de flacidez abdominal através da eletroacupuntura é realizado utilizando pontos motores localizados na área abdominal, buscando tonicidade para essa área (NAKANO e YAMAMURA, 2005).

Para o tratamento de flacidez do braço é utilizado eletroacupuntura de alta frequência em torno de $300 \mathrm{~Hz}$, porém, em seguida, é necessário tonificar com frequência 2 a $10 \mathrm{~Hz}$ os meridianos 


\section{FAZ CIÊNCIA, VOL. 23, N. 37, JAN/JUN DE 2021 - P. 211 - 224}

localizados na área de flacidez, também se faz necessário estimular os pontos motores encontrados no percurso do meridiano (NAKANO e YAMAMURA, 2005).

A eletroacupuntura também pode ser utilizada para o tratamento de flacidez da perna, são estimulados os pontos motores localizados na região de flacidez, também podem ser utilizados pontos de acupuntura localizados na perna (NAKANO e YAMAMURA, 2005).

\section{Microagulhamento}

O microagulhamento é uma técnica inovadora e pode ser empregue para diversos tratamentos que possuem como objetivo o estímulo da produção de colágeno (LIMA, LIMA e TAKANO, 2013).

Do ponto de vista energético, o microagulhamento promove circulação de Qi, Xue e Jing Ye (FERNANDES, 2015).

Benefícios do tratamento com microagulhamento na estética:

- $\quad$ Produção de colágeno;

- $\quad$ Promove viscosidade a pele;

- $\quad$ Reduz rugas e linhas de expressão;

- $\quad$ Reduz cicatriz de acne;

- $\quad$ Reduz celulite;

- $\quad$ Reduz estrias;

- $\quad$ Reduz flacidez.

Para a realização da técnica de microagulhamento, sugere-se colocar o aparelho utilizado para o microagulhamento entre os dedos indicador e polegar, é necessário ter cuidado com a força do polegar. São realizados movimentos de vai e vem, que devem realizar um padrão semelhante de petéquias em toda a região em que está sendo realizado o procedimento. É necessário realizar em torno de 10 e 15 passadas do aparelho na mesma direção e percorrer pelo menos quatro sentidos nas regiões tratadas (LIMA, LIMA e TAKANO, 2013).

Segundo Fernandes (2015), o tratamento deve respeitar intervalos, que depende do ciclo de produção do novo colágeno, que acontece por volta de vinte e um a vinte e oito dias em procedimentos de pressão forte, fazendo com que o tratamento aconteça uma vez ao mês.

Para o tratamento de estrias é indicado aplicar analgésico para fazer com que o procedimento fique mais confortável. Deve ser realizado o procedimento com agulhas de 1,5 ou 


\section{FAZ CIÊNCIA, VOL. 23, N. 37, JAN/JUN DE 2021 - P. 211 - 224}

2,0 $\mathrm{mm}$, aplicar o aparelho no tempo de 10 minutos percorrendo os quatro sentidos. No final do procedimento pode ser utilizado óleo de semente de uva ou copaíba (FERNANDES, 2015).

No tratamento de celulite é indicado utilizar agulhas de 1,5 ou 2,0 mm, quando se trata de celulite grau 1 e 2, enquanto que para celulite grau 3 é aconselhado utilizar agulha de 3,0 mm. O procedimento deve durar em torno de 10 a 15 minutos nos quatro sentidos. Para finalizar pode ser utilizada ventosa, dando prioridade a ventosa com pistola, fazendo uso no sentido do joelho para as nádegas (FERNANDES, 2015).

No tratamento de rugas e linhas de expressão o tamanho da agulha vai depender do tipo de pele, podendo variar de 0,2 a 3,0 mm. Para marcas de acnes são indicadas agulhas de tamanhos maiores, por exemplo, 2,0 e 3,0 mm (FERNANDES, 2015).

Segundo Fernandes (2015), após o procedimento o paciente deve ser orientado a não se expor ao sol e utilizar protetor solar de no mínimo fator 30. Quando o procedimento for realizado no rosto, orientar o paciente a não fazer uso de maquiagem por pelo menos 24 horas.

\section{Resultados e discussão}

Segundo os artigos pesquisados para elaboração desse trabalho podemos dizer que as práticas da MTC, além de serem muito conhecidas para tratar patologias, também conquistam bons resultados na estética.

O tratamento estético utilizando as práticas da MTC é realizado do mesmo modo que sessões de acupuntura para tratar doenças. $\mathrm{O}$ tratamento busca trabalhar nos pontos de acupuntura para obter equilíbrio energético no organismo, desta forma, equilibra os órgãos internos resultando em benefícios para a parte externa do corpo, o tratamento atua na queixa estética do paciente sendo um tratamento duradouro, pois trata o paciente de dentro para fora.

O tratamento utilizando as práticas da MTC deve ser realizado por um profissional competente e habilitado, pois será necessário avaliar o paciente, analisar sua queixa estética e realizar um tratamento verificando o que está em desequilíbrio no seu organismo e utilizar as práticas da MTC mais adequadas para a queixa estética que o paciente deseja melhorar.

O tratamento pode ser realizado em todas as idades, são práticas pouco invasivas ou em alguns casos nada invasivas e possui raras contraindicações.

Com base nos autores estudados, foi desenvolvido um quadro (Quadro 1) com os resultados alcançados na pesquisa sobre as práticas da MTC na estética, onde foram demonstrados quais as práticas da MTC são mais eficazes para redução de manchas na pele, rugas, gordura localizada, celulite, flacidez, estrias, cicatriz de acne, produção de colágeno, produção de viço a pele, hidratação da pele e emagrecimento. 
Quadro 1 - Resultados da revisão de literatura sobre as práticas da Medicina Tradicional Chinesa na estética

\begin{tabular}{|c|c|c|c|c|}
\hline AUTOR & $\begin{array}{l}\text { TITULO DO } \\
\text { TRABALHO }\end{array}$ & ANO & PRÁTICA DA MTC & RESULTADOS \\
\hline FERNANDES, F.A.C. & $\begin{array}{c}\text { Acupuntura Estética } \\
\text { - Prática e Objetiva: } \\
\text { Novos } \\
\text { Procedimentos }\end{array}$ & 2015 & $\begin{array}{c}\text { Pó de pérola } \\
\text { Algas marinhas }\end{array}$ & $\begin{array}{c}\text { Redução de } \\
\text { manchas na pele. }\end{array}$ \\
\hline FERNANDES, F.A.C. & $\begin{array}{c}\text { Acupuntura Estética } \\
\text { - Prática e Objetiva: } \\
\text { Novos } \\
\text { Procedimentos }\end{array}$ & 2015 & $\begin{array}{c}\text { Microagulhamento } \\
\text { Moxaterapia }\end{array}$ & \multirow{4}{*}{ Redução de rugas. } \\
\hline $\begin{array}{l}\text { NAKANO, M.A.Y; } \\
\text { YAMAMURA, Y. }\end{array}$ & $\begin{array}{c}\text { Acupuntura em } \\
\text { Dermatologia e } \\
\text { Medicina Estética }\end{array}$ & 2005 & Eletroacupuntura & \\
\hline AIABE, R. & $\begin{array}{l}\text { Acupuntura estética } \\
\text { e acupuntura a laser } \\
\text { auxiliam no } \\
\text { emagrecimento }\end{array}$ & 2011 & Laser & \\
\hline RINALDI, A. & $\begin{array}{l}\text { Acupuntura estética } \\
\text { corporal e facial }\end{array}$ & 2014 & Acupuntura & \\
\hline FERNANDES, F.A.C. & $\begin{array}{c}\text { Acupuntura Estética } \\
\text { - Prática e Objetiva: } \\
\text { Novos } \\
\text { Procedimentos }\end{array}$ & 2015 & $\begin{array}{c}\text { Moxaterapia } \\
\text { Guasha } \\
\text { Algas marinhas } \\
\text { Pó de pérola } \\
\text { Microagulhamento }\end{array}$ & $\begin{array}{l}\text { Produção de } \\
\text { colágeno. }\end{array}$ \\
\hline FERNANDES, F.A.C. & $\begin{array}{c}\text { Acupuntura Estética } \\
\text { - Prática e Objetiva: } \\
\text { Novos } \\
\text { Procedimentos }\end{array}$ & 2015 & $\begin{array}{c}\text { Moxaterapia } \\
\text { Guasha } \\
\text { Microagulhamento }\end{array}$ & $\begin{array}{l}\text { Promove } \\
\text { viscosidade a pele. }\end{array}$ \\
\hline $\begin{array}{l}\text { NAKANO, M.A.Y; } \\
\text { YAMAMURA, Y. }\end{array}$ & $\begin{array}{l}\text { Acupuntura em } \\
\text { Dermatologia e } \\
\text { Medicina Estética }\end{array}$ & 2005 & Eletroacupuntura & \\
\hline
\end{tabular}


FAZ CIÊNCIA, VOL. 23, N. 37, JAN/JUN DE 2021 - P. 211 - 224

\begin{tabular}{|c|c|c|c|c|}
\hline AIABE, R. & $\begin{array}{c}\text { Acupuntura estética } \\
\text { e acupuntura a laser } \\
\text { auxiliam no } \\
\text { emagrecimento }\end{array}$ & 2011 & Laser & $\begin{array}{c}\text { Redução de } \\
\text { gordura localizada. }\end{array}$ \\
\hline FORNAZIERI, L.C. & $\begin{array}{c}\text { Tratado de } \\
\text { Acupuntura Estética }\end{array}$ & 2007 & Ventosa & \\
\hline RINALDI, A. & $\begin{array}{c}\text { Acupuntura estética } \\
\text { corporal e facial }\end{array}$ & 2014 & Acupuntura & \\
\hline FERNANDES, F.A.C. & $\begin{array}{c}\text { Acupuntura Estética } \\
\text { - Prática e Objetiva: } \\
\text { Novos } \\
\text { Procedimentos }\end{array}$ & 2015 & Microagulhamento & \multirow{5}{*}{$\begin{array}{l}\text { Redução de } \\
\text { celulite. }\end{array}$} \\
\hline $\begin{array}{l}\text { NAKANO, M.A.Y; } \\
\text { YAMAMURA, Y. }\end{array}$ & $\begin{array}{l}\text { Acupuntura em } \\
\text { Dermatologia e } \\
\text { Medicina Estética }\end{array}$ & 2005 & Eletroacupuntura & \\
\hline AIABE, R. & $\begin{array}{c}\text { Acupuntura estética } \\
\text { e acupuntura a laser } \\
\text { auxiliam no } \\
\text { emagrecimento }\end{array}$ & 2011 & Laser & \\
\hline FORNAZIERI, L.C. & $\begin{array}{c}\text { Tratado de } \\
\text { Acupuntura Estética }\end{array}$ & 2007 & Ventosa & \\
\hline RINALDI, A. & $\begin{array}{c}\text { Acupuntura estética } \\
\text { corporal e facial }\end{array}$ & 2014 & Acupuntura & \\
\hline FERNANDES, F.A.C. & $\begin{array}{c}\text { Acupuntura Estética } \\
\text { - Prática e Objetiva: } \\
\text { Novos } \\
\text { Procedimentos }\end{array}$ & 2015 & Microagulhamento & \multirow{3}{*}{$\begin{array}{l}\text { Redução de } \\
\text { flacidez. }\end{array}$} \\
\hline $\begin{array}{l}\text { NAKANO, M.A.Y; } \\
\text { YAMAMURA, Y. }\end{array}$ & $\begin{array}{c}\text { Acupuntura em } \\
\text { Dermatologia e } \\
\text { Medicina Estética }\end{array}$ & 2005 & Eletroacupuntura & \\
\hline AIABE, R. & $\begin{array}{l}\text { Acupuntura estética } \\
\text { e acupuntura a laser }\end{array}$ & 2011 & Laser & \\
\hline
\end{tabular}




\begin{tabular}{|c|c|c|c|c|}
\hline & $\begin{array}{l}\text { auxiliam no } \\
\text { emagrecimento }\end{array}$ & & & \\
\hline RINALDI, A. & $\begin{array}{c}\text { Acupuntura estética } \\
\text { corporal e facial }\end{array}$ & 2014 & Acupuntura & \\
\hline FERNANDES, F.A.C. & $\begin{array}{c}\text { Acupuntura Estética } \\
\text { - Prática e Objetiva: } \\
\text { Novos } \\
\text { Procedimentos }\end{array}$ & 2015 & Microagulhamento & \multirow{2}{*}{ Redução de estrias. } \\
\hline $\begin{array}{l}\text { NAKANO, M.A.Y; } \\
\text { YAMAMURA, Y. }\end{array}$ & $\begin{array}{l}\text { Acupuntura em } \\
\text { Dermatologia e } \\
\text { Medicina Estética }\end{array}$ & 2005 & Eletroacupuntura & \\
\hline FERNANDES, F.A.C. & $\begin{array}{c}\text { Acupuntura Estética } \\
\text { - Prática e Objetiva: } \\
\text { Novos } \\
\text { Procedimentos }\end{array}$ & 2015 & Microagulhamento & $\begin{array}{l}\text { Redução de cicatriz } \\
\text { de acne. }\end{array}$ \\
\hline FERNANDES, F.A.C. & $\begin{array}{c}\text { Acupuntura Estética } \\
\text { - Prática e Objetiva: } \\
\text { Novos } \\
\text { Procedimentos }\end{array}$ & 2015 & $\begin{array}{l}\text { Algas marinhas } \\
\text { Pó de pérola }\end{array}$ & Hidratação da pele. \\
\hline AIABE, R. & $\begin{array}{c}\text { Acupuntura estética } \\
\text { e acupuntura a laser } \\
\text { auxiliam no } \\
\text { emagrecimento }\end{array}$ & 2011 & Laser & \multirow{3}{*}{ Emagrecimento. } \\
\hline DOMINGO, S.J.J. & Auriculoterapia & 2011 & Auriculoterapia & \\
\hline RINALDI, A. & $\begin{array}{l}\text { Acupuntura estética } \\
\text { corporal e facial }\end{array}$ & 2014 & Acupuntura & \\
\hline
\end{tabular}

Fonte: As autoras (2021).

\section{Conclusão}

Conclui-se que as práticas da MTC abordadas no trabalho já são muito conhecidas para o tratamento de patologias e são muito utilizadas em vários países, porém, as práticas da MTC vêm ganhando espaço na área da estética e estão conseguindo alcançar resultados satisfatórios.

Um ponto importante que vale salientar, é que são práticas que na estética não buscam somente apresentar bons resultados na aparência externa do paciente, as práticas da MTC buscam equilíbrio energético do organismo do paciente, equilibram os órgãos internos para em seguida serem manifestados bons resultados na queixa estética do paciente. 
O tratamento utilizando as práticas da MTC possui muitas vantagens, são práticas pouco ou nada invasivas, baixo risco de alergia, raras contraindicações e podem ser utilizadas em todas as idades.

\section{Referências}

AIABE, R. Acupuntura estética e acupuntura a laser auxiliam no emagrecimento. Corpo em harmonia blogspot, 30 Out. 2011. Disponível em: http://robertoaiabemassoterapeuta.blogspot.com.br/2011/10/acupuntura-estetica-auxilia-no.html Acesso em: 28 mar. 2021.

ALMEIDA-LOPES, L. Laserpuntura: Bases Científicas e Aplicações - Revisão Bibliográfica. São Carlos. Monografia (Especialização em Medicina Tradicional Chinesa). Escola Spaço Alternativo. 2010.

ANDRÉ, K. C. O Tratamento da Dor com Laser Acupuntura. Mogi das Cruzes. Monografia (Especialização em Acupuntura). Universidade Mogi das Cruzes. 2011.

BRASIL. Ministério da Saúde. Política Nacional de Práticas Integrativas e Complementares no SUS: PNPIC: atitude de ampliação de acesso. Brasília, DF, 2006. Disponível em: http://bvsms.saude.gov.br/bvs/publicacoes/pnpic.pdf Acesso em: 28 mar. 2021.

CUNHA, A. A. Ventosaterapia. São Paulo: Ícone, 1996.

DAL-MAS, W. D. Auriculoterapia - Auriculomedicina na Doutrina Brasileira. São Paulo: Roca, 2004.

DOMINGO, S. J. J. Auriculoterapia. Matinhos Universidade Federal do Paraná, Matinhos, 2011.

FERNANDES, F. A. C. Acupuntura Estética - Prática e Objetiva: Novos Procedimentos. 2. ed. São Paulo: Ícone, 2015.

FLEISCHMAN, G. F. Acupuntura - Tudo o que você sempre quis saber. São Paulo: Madras, 2000 .

FORNAZIERI, L. C. Tratado de Acupuntura Estética. 2. ed. São Paulo: Ícone, 2007.

LIMA, E.V.A.; LIMA, M.A.; TAKANO, D. Microagulhamento: estudo experimental e classificação da injúria provocada. Surgical \& Cosmetic Dermatology, vol. 5, n. 2, p. 110-114, 2013.

MANN, F. Acupuntura: A arte chinesa de curar. São Paulo: Hemus, 1994.

MIN, L. S.; DARELlA, M. L.; PEREIRA, O. A. A. Curso Básico - Acupuntura e Medicina Tradicional Chinesa. Florianópolis: IPE-MTC, 2000.

NAKANO, A. A. Y; YAMAMURA, Y. Acupuntura em Dermatologia e Medicina Estética. São Paulo: Livraria Médica Paulista Editora, 2005.

RINALDI, A. Acupuntura estética corporal e facial. São Paulo, 2014. Disponível em: http://www.gazetavirtual.com.br/acupuntura-estetica-corporal-e-facial Acesso em: 28 mar. 2021.

ROSS, J. Zang Fu - Sistemas de Órgãos e Vísceras da Medicina Tradicional Chinesa. 2. ed. São Paulo: Roca, 1994. 
SOUSA, L.; HADDAD, M. L.; NAKANO, M. A. S.; GOMES, F. A. Terapêutica nãofarmacológica para alívio do ingurgitamento mamário durante a lactação: revisão integrativa da literatura. Revista da Escola de Enfermagem da USP, São Paulo, vol. 46, n. 2, p. 472-479, abril, 2012.

WEN, T. S. Acupuntura Clássica Chinesa. São Paulo: Cultrix, 1985.

YAMAMURA, Y. Acupuntura Tradicional: A Arte de Inserir. 2. ed. São Paulo: Roca, 2004. . Acupuntura - Um texto compreensível. São Paulo: Roca, 1996.

Recebido em 05/05/2020 - Aprovado em 19/12/2020 